\title{
Physical Activity and Sleep Analysis of Heart Failure Patients using Multi-sensor Patches
}

\author{
Johanna O'Donnell ${ }^{1,2}$, Carmelo Velardo ${ }^{1}$, Syed Ahmar Shah ${ }^{3}$, Gholamreza Salimi Khorshidi ${ }^{2}$, \\ Dario Salvi ${ }^{1}$, Kazem Rahimi ${ }^{2}$, Lionel Tarassenko ${ }^{1}$
}

\begin{abstract}
Traditional heart failure markers fail to reliably predict heart-failure related hospitalisations and deaths. Multisensor patch data can provide an objective insight into activity and sleep patterns of patients and may therefore improve the performance of current risk-quantification algorithms. This work aimed to establish the feasibility of collecting multi-sensor patch data from heart failure patients and to perform an initial analysis of activity and sleep patterns of heart failure patients in relation to disease severity. 13 heart failure patients from the SUPPORT-HF study were provided with chest-worn multisensor patches and asked to wear the devices continuously for up to seven consecutive days. Using a combination of impedance, heart rate and accelerometer data participants' sleep and wakefulness information were extracted and analyzed in relation to self-reported symptom scores. Patch data for eleven patients were of high enough quality to be included in the analysis, accounting for 63 patient days worth of data. The heart failure patients slept for an average of 8.3 hours a night and experienced 2.8 sleep interruptions. Potential differences in sleep angle, heart rate and wake-time activity were found for patients with different heart failure severity. Larger studies are necessary to create a more coherent picture of the potential of activity and sleep as a markers for heart failure deterioration.
\end{abstract}

Index Terms-Multi-sensor, Accelerometer, Activity, Sleep, Heart Failure

\section{INTRODUCTION}

Heart failure is a chronic condition that affects approximately 900,000 people in the UK [1]. It is estimated that nearly $20 \%$ of patients diagnosed with the condition die within one year of diagnosis [2]. Continuous monitoring may allow for an early identification of disease deterioration and therefore increase patients' likelihood of survival [3], however current non-invasive markers for continuous monitoring fail to accurately predict heart-failure related hospitalisations and deaths [4].

Physical activity and sleep behaviors of heart failure patients may offer an insight into patients' well-being and provide new markers to predict heart failure deterioration. In previous

The authors acknowledge the support of the NIHR Oxford BRC grant. Thanks go to the patients who accepted to take part in this research. Thanks also go to Dr. Tracey Chantler, Dr. Chris Paton and Emma Stoppani for the data collection. J. O'Donnell acknowledges the support of the RCUK Digital Economy Programme grant number EP/G036861/1 (Oxford Centre for Doctoral Training in Healthcare Innovation)

${ }^{1}$ J. O'Donnell, C. Velardo, D. Salvi and L. Tarassenko are with the Institute of Biomedical Engineering, Department of Engineering Science, University of Oxford, Oxford, UK

${ }^{2}$ R. Salimi Khorshidi and K. Rahimi are with the George Institute for Global Health, University of Oxford, Oxford, UK

${ }^{2}$ S.A. Shah is with the MRC Brain Network Dynamics Unit and Nuffield Department of Clinical Neurosciences, University of Oxford, UK studies, low accelerometer-derived physical activity levels measured over short periods of time (e.g. one-week) have been linked to an increased risk of long-term mortality and hospitalisation [5] [6]. In addition to this, sleep conditions such as sleep apnea have been shown to have a negative impact on heart failure patients' quality of life and potentially affect their likelihood of survival [7]. Research on the shortterm predictive value of physical activity and sleep behaviors has however been limited.

Multi-sensor patches containing accelerometer sensors can provide an objective tool to monitor physical activity and sleep behaviors of patients over time [8]. They also offer to link these data to other physiological measurements, such as heart rate information. As a result, they have the potential to help understand the impact of disease on patient lifestyle and offer a means for short-term risk-quantification. The purpose of this research was to evaluate the feasibility of using multisensor patches for heart failure monitoring and to gain an initial insight into activity and sleep patterns of heart failure patients with a focus on how these differ across patients with different heart failure severities.

\section{METHOdS}

\section{A. Data Collection}

Data were collected as part of the SUPPORT-HF study [9]. The aim of the study was to develop and evaluate a user-centered home monitoring system for heart failure patients. Patients with a confirmed diagnosis of heart failure were eligible to take part in the study. After providing informed consent, they were asked to record daily weight and blood pressure readings, and to self-assess their New York Heart Failure Association (NYHA) scores daily for a period of six months. The NYHA score ranks heart failure severity on a scale between 1 (lowest severity) to 4 (highest severity) based on self-reported breathlessness during rest and physical activity. A subset of 13 patients, selected so to include patients with a range of NYHA scores, was asked to wear a multi-sensor chest-worn patch for as long as they could (up to a maximum of seven days). Approval for the SUPPORT-HF study was obtained from the NHS Health Research Authority (NRES Committee South CentralOxford, reference B13/SC/0125). 


\section{B. Device}

The Proteus patch (Proteus Digital Health, Redwood City, USA) is a FDA-approved multi-sensor device designed to monitor physiological, movement and medication adherence data of patients. It is waterproof and equipped with a temperature sensor, tri-axial accelerometer and two electrodes capable of recording skin impedance and heart rate data [10]. The chest-worn device was chosen for the SUPPORTHF study due to its ability to record heart rate as well as accelerometer data and to detect body position using the chest accelerometer data.

The 13 patients who agreed to take part in the data collection were visited by a research team consisting of an engineer and a qualitative researcher. They were instructed to apply the patch to their upper left chest, on the side of the rib-cage and were allowed to choose the exact orientation of the device so to minimize discomfort. Patients applied the patches themselves with only limited assistance from the researchers. They were asked to wear the devices continuously for the duration of the recording and were provided with a prepaid envelope to post the patch back to the researcher team after recording.

The multi-sensor patch was set to record tri-axial accelerometer data at a sampling frequency of $5 \mathrm{~Hz}$. Based on the developer settings, data from the first 15 seconds of each minute were aggregated (using the average) into one-minute epochs. Temperature and skin impedance measurements were taken every five and ten minutes respectively; heart rate summary statistics including the mean heart rate and RR interval length were measured during 12 seconds of each ten-minute epoch.

\section{Data Processing}

1) Wear-time Detection:: Using the device-internal skinimpedance sensors, wear and non-wear periods were identified; As the difference in impedance between air and the electrode surface is much larger than the difference in impedance between skin and the electrode surface, smaller impedance values were expected when the patch is worn.

Experimental analysis of the impedance of the patch during wear and non-wear periods showed a mean value of 1.2 $\mathrm{k} \Omega$ during wear times and approximately $4.2 \mathrm{k} \Omega$ during non-wear times. Based on these values and the manufacturers recommendations, a wear-threshold of $4 \mathrm{k} \Omega$ was chosen to differentiate between the two states. Whenever the impedance measured by both impedance sensors $\left(R 1_{n}\right.$ or $R 2_{n}$ ) exceeded $4 \mathrm{k} \Omega$, the device was considered not to be worn $\left(w_{n}=0\right)$; Otherwise it was considered to be worn $\left(w_{n}\right.$ =1) (Equation 1).

$$
w_{n}= \begin{cases}1, & \text { if } R 1_{n}<4 \mathrm{k} \Omega \text { or } R 2_{n}<4 \mathrm{k} \Omega \\ 0, & \text { otherwise }\end{cases}
$$

Patients with less than two days of wear-time data were identified and removed from the analysis.

2) Sleep Detection: Sleep-detection algorithms for wristworn accelerometers are based on the identification of sustained periods of low movement [11]. Well-validated methods include the weighted-sum approach by Cole et al. [12] and the idleness-identifying method by Borazio et al. [11]. These methods are based on acceleration along the palmar-dorsal direction, which is difficult to locate from a self-applied chest-worn accelerometer. In addition to this, the acceleration experienced by the torso-worn patch during movement is likely to be reduced in comparison to that experienced by wrist-worn devices, which could result in an overestimation of sleep times.

In order to create an estimate of sleep and wakefulness periods using the chest-worn device, body positioning $\left(p_{n}\right)$ information was used. Angular information $\left(a_{n}\right)$ in the vertical direction (along the $\mathrm{x}$-axis of the patch) was retrieved using Equation 2, where $x_{n}, y_{n}, z_{n}$ are the accelerations at sample $n$ along the $x, y$ and $z$ axis. $90^{\circ}$ corresponded to a flat lying position and $0^{\circ}$ to an upright sitting or standing position.

$$
a_{n}=\operatorname{atan}\left(\frac{x_{n}}{\sqrt{y_{n}^{2}+z_{n}^{2}}}\right) \frac{180}{\pi} .
$$

A threshold of $60^{\circ}$ was chosen, where angles below $60^{\circ}$ were classed as corresponding to non-lying positions and angles above $60^{\circ}$ as corresponding to lying positions.

$$
p_{n}= \begin{cases}\text { lying, } & \text { if } a_{n} \geq 60^{\circ} \\ \text { upright, } & \text { otherwise }\end{cases}
$$

In order to remove the effect of short-term wake/ upright periods during sleep and short-term sleep/ lying periods during wake-times, a mean-filter of filter length $\mathrm{N}=60$ minutes was applied to the position labels. 60 minutes was chosen based on experimental findings on the SUPPORTHF data itself that suggested that this filter length was successful at removing short periods of lying during waketimes and vice versa. Future research should validate this threshold using self-reported sleep onset and wake-up times as reference.

\section{Analysis}

Heart rate, temperature, the standard deviation of the vector magnitude of one-minute epoch acceleration (vmag_std) and angular data were extracted for each patient and studied in relation to self-reported heart failure severity. Latter was defined according to the NYHA classification score. Where available, NYHA scores during the period of patch recording were utilized. Where this was not possible, the mean NYHA value across the SHF study-period was taken as reference. 

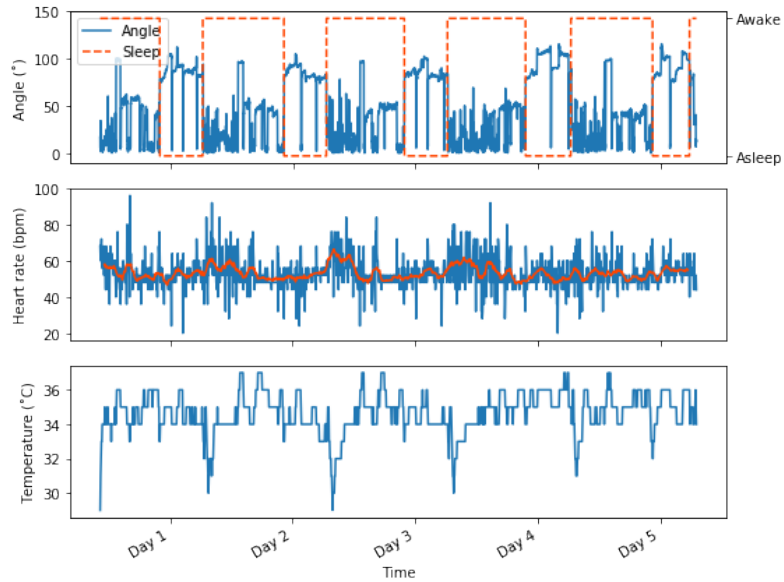

Fig. 1. Example signals collected from patch. Top to bottom: Angular data (blue solid line) and derived sleep and wake labels (orange dashed line), heart rate (solid blue line) and smoothed heart rate for visualization (orange solid line), and temperature data over time.

Mean and confidence intervals for each of the variables were recorded during sleep and wake periods and presented stratified by heart failure severity.

\section{RESULTS}

Out of 13 patients provided with the multi-sensor patches, two patients had to be removed from the analysis due to insufficient or noisy data. One of these patients was the only patient with a NYHA score of 4 . This patient had such noisy accelerometer data that sleep and wake periods could not be extracted. The remaining eleven patients had a mean (SD) age of 79 (8.3) years; four of them were male and seven were female. Two patients had an NYHA score of 1, seven an NYHA score of 2 and two an NYHA score of 3. Table I summarizes the patients' baseline characteristics stratified by NYHA class.

TABLE I

BASELINE CHARACTERISTICS. * MEAN (SD)

\begin{tabular}{llll}
\hline & NYHA $=\mathbf{1}$ & NYHA = 2 & NYHA = 3 \\
\hline $\mathrm{N}$ & 2 & 7 & 2 \\
Females & $100 \%$ & $43 \%$ & $0 \%$ \\
Age $^{*}$ & $85(0.7)$ & $77(9.9)$ & $78(2.8)$ \\
\hline
\end{tabular}

The participants wore the patch for an average of 5.7 days (standard deviation $(\mathrm{SD})=1.3$ days). The average total sleep time across all participants was 8.3 hours ( $\mathrm{SD}=1.2$ hours) and the average number of sleep interruptions was 2.8 (SD $=1.2$ ). Figure 1 provides example traces of the different signals extracted from the chest-worn patch as well as the sleep labels derived using the angular-data approach.

Table II summarizes the extracted variables stratified by NYHA class. Looking at the sleep markers, it can be seen that patients with a NYHA of 3 slept at a lower angle with respect to the standing position (i.e. they slept in a more upright position) than patients with lower NYHA values. In addition to this, their movement (measured using vmag_std) during
TABLE II

SLEEP AND WAKEFULNESS CHARACTERISTICS STRATIFIED BY NYHA

\begin{tabular}{llll}
\hline & $\begin{array}{l}\text { NYHA = 1 } \\
\text { Mean (SD) }\end{array}$ & $\begin{array}{l}\text { NYHA = 2 } \\
\text { Mean (SD) }\end{array}$ & $\begin{array}{l}\text { NYHA = 3 } \\
\text { Mean (SD) }\end{array}$ \\
\hline $\begin{array}{l}\text { Days } \\
\text { Total sleep }\end{array}$ & $8.6(1.4)$ & $5.4(1.3)$ & $5.5(0.7)$ \\
time (h) & $8.0(1.3)$ & $8.9(0.7)$ \\
$\begin{array}{l}\text { Sleep angle }\left(^{\circ}\right) \\
\text { Sleep tempera- }\end{array}$ & $83.0(1.1)$ & $84.3(4.5)$ & $78.4(1.9)$ \\
ture $\left({ }^{\circ} \mathrm{C}\right)$ & $35.6(0.3)$ & $35.0(0.6)$ & $34.8(0.4)$ \\
$\begin{array}{l}\text { Sleep heart rate } \\
\text { (bpm) }\end{array}$ & $38.8(6.5)$ & $56.2(12.4)$ & $63.2(3.0)$ \\
$\begin{array}{l}\text { Sleep } \\
\text { vmag_std } \\
\text { (mg) }\end{array}$ & $25(0.8)$ & $25(1.4)$ & $33(0.3)$ \\
$\begin{array}{l}\text { Sleep Interrup- } \\
\text { tions (n) }\end{array}$ & $5.5(3.9)$ & $2.5(1.2)$ & $2.5(2.4)$ \\
\hline $\begin{array}{l}\text { Wake angle }\left({ }^{\circ}\right) \\
\text { Wake tempera- } \\
\text { ture }\left({ }^{\circ} \mathrm{C}\right)\end{array}$ & $29.8(4.7)$ & $28.2(6.1)$ & $25.9(5.7)$ \\
$\begin{array}{l}\text { Wake heart rate } \\
\text { bpm) }\end{array}$ & $45.5(0.0)$ & $63.3(0.8)$ & $33.7(0.3)$ \\
$\begin{array}{l}\text { Wake } \\
\text { vmag_std } \\
\text { (mg) }\end{array}$ & $36(0.5)$ & $39(0.1)$ & $32(0.0)$ \\
$\begin{array}{l}\text { Wake interrup- } \\
\text { tions/ naps (n) }\end{array}$ & $1(0.5)$ & $0.4(0.8)$ & $0.1(0.4)$ \\
\hline
\end{tabular}

sleep was higher, potentially suggesting more disrupted sleep patterns. The number of sleep interruptions was highest in the group of patients with an NYHA of 1, due to the fact that one of the two patients with an NYHA of 1 experienced very frequent interruptions.

During both sleep and wake-times, an increased heart rate seemed to be associated with more severe heart failure. Temperatures were similar across patients with different NYHA scores and the patients with lower NYHA scores were more prone to day-time napping. Finally, the amount of day-time activity (quantified using vmag_std) decreased with heart failure severity.

\section{Discussion AND CONCLUSION}

We acquired and analyzed multi-sensor patch data from heart failure patients with different heart failure severities. The analysis showed that it is feasible to extract sleep and wake time patterns of heart failure patients using a chestworn device. Patients were willing to wear the devices for a multi-day period, despite the fact that they were relatively rigid and the adhesive material was non-ideal. Since the data collection, a newer, optimized version of the patch has been developed. In addition to this, other devices, such as the multi-sensor VitalPatch [13], have been developed to gather similar long-term data.

Clean signals could be extracted for most patients; however the only patient included in the analysis with an NYHA of 4 had very noisy accelerometer readings and sleep and wake 
information could not be extracted. Whilst this may be due to poor placement of the patch, it could also be down to unusual sleep and wake behavior, e.g. large proportions of the day spent lying. Further research should focus on the feasibility of monitoring activity patterns of patients with very severe forms of the condition, as well as provide a validation and evaluation of the quality of the signals collected by the device (e.g. heart rate and sleep-wake cycles).

The analysis on eleven heart failure patients found that patients with more severe heart failure were less physically active during the day (smaller vmag_std values). This finding is in agreement with previous findings by Dontje et al. [14] who studied the activity patterns of 68 heart failure patients over 48 hours and found that higher NYHA classifications were associated with lower physical activity levels. In addition to this, sleep angles seemed to vary across patients with different heart failure severity, with patients with more severe heart failure sleeping in a more upright position. This may be related to findings by Basoglu et al. [15], who suggested that a raised sleeping position (e.g. through use of additional pillows) may relieve symptoms of sleep apnea in heart failure patients. Both vmag_std and sleep angle may be suitable markers for future heartfailure risk-quantification algorithms. Finally, patients with increased NYHA scores had higher heart rates both during sleep and wakefulness, which has previously been identified as a risk marker for heart failure deterioration [16].

Limitations of this analysis include the fact that the findings discussed in this analysis are based on a very small sample of heart failure patients. The number of patients in each NYHA group were different with most patients included in the study having a NYHA score of 2 . As a results, differences between groups were not significant and findings may not be representable of the larger heart failure population.

For the purpose of this analysis, body positioning information was used to extract sleep and wake information from the chest-adherent patch. This approach is different to standard accelerometer-based methods, which mostly rely on the identification of sustained periods of low movement. However the wearing location of the device made positioning-based sleep detection more suitable. In future, body position information should be adjusted for wear-angle.

Despite the limitations, our pilot gathered data from eleven out of 13 patients. It demonstrates the feasibility of using smart patches to extract multi-sensor, high frequency data from chronically ill patients. In addition to this, our results suggest that both physical activity and sleep data may vary across patients with different heart failure severities and may provide suitable markers for patient deterioration. Larger studies are necessary to further evaluate these markers.

\section{REFERENCES}

[1] N. Conrad, A. Judge, J. Tran, H. Mohseni, D. Hedgecott, A. P. Crespillo, M. Allison, H. Hemingway, J. G. Cleland, J. J. V. McMur- ray, and K. Rahimi, "Temporal trends and patterns in heart failure incidence: a population-based study of 4 million individuals," The Lancet, Nov. 2017.

[2] C. J. Taylor, R. Ryan, L. Nichols, N. Gale, F. R. Hobbs, and T. Marshall, "Survival following a diagnosis of heart failure in primary care," Family Practice, vol. 34, pp. 161-168, Apr. 2017.

[3] V. Konstam, D. Gregory, J. Chen, A. Weintraub, A. Patel, D. Levine, D. Venesy, K. Perry, C. Delano, and M. A. Konstam, "Health-Related Quality of Life in a Multicenter Randomized Controlled Comparison of Telephonic Disease Management and Automated Home Monitoring in Patients Recently Hospitalized With Heart Failure: SPAN-CHF II Trial," Journal of Cardiac Failure, vol. 17, pp. 151-157, Feb. 2011.

[4] K. McDonald, M. Wilkinson, and M. Ledwidge, "Role of Monitoring Devices in Preventing Heart Failure Admissions," Current Heart Failure Reports, vol. 12, pp. 269-275, Aug. 2015.

[5] K. P. Izawa, S. Watanabe, K. Oka, K. Hiraki, Y. Morio, Y. Kasahara, P. H. Brubaker, N. Osada, K. Omiya, and H. Shimizu, "Usefulness of Step Counts to Predict Mortality in Japanese Patients With Heart Failure," The American Journal of Cardiology, vol. 111, pp. 17671771, June 2013.

[6] E. Rullman and T. Gustafsson, "Physical Activity Assessed with Accelerometer is an Accurate and Independent Predictor of Mortality in Patients with Advanced Heart Failure," The FASEB Journal, vol. 29, p. LB598, Apr. 2015.

[7] S. Javaheri, R. Shukla, H. Zeigler, and L. Wexler, "Central sleep apnea, right ventricular dysfunction, and low diastolic blood pressure are predictors of mortality in systolic heart failure," Journal of the American College of Cardiology, vol. 49, pp. 2028-2034, May 2007.

[8] G. Spina, M. A. Spruit, J. Alison, R. P. Benzo, A. C. d. Brinker, and et al., "Analysis of nocturnal actigraphic sleep measures in patients with COPD and their association with daytime physical activity," Thorax, Feb. 2017.

[9] K. Rahimi, C. Velardo, A. Triantafyllidis, N. Conrad, S. A. Shah, T. Chantler, H. Mohseni, E. Stoppani, F. Moore, C. Paton, C. A. Emdin, J. Ernst, and L. Tarassenko, "A user-centred home monitoring and self-management system for patients with heart failure: A multicentre cohort study," European Heart Journal - Quality of Care and Clinical Outcomes, p. qcv013, July 2015.

[10] H. Hafezi, T. Robertson, G. Moon, K. Au-Yeung, M. Zdeblick, and G. Savage, "An Ingestible Sensor for Measuring Medication Adherence," IEEE Transactions on Biomedical Engineering, vol. 62, pp. 99-109, Jan. 2015.

[11] M. Borazio, E. Berlin, N. Kuecuekyildiz, P. Scholl, and K. V. Laerhoven, "Towards Benchmarked Sleep Detection with Wrist-Worn Sensing Units," in 2014 IEEE International Conference on Healthcare Informatics (ICHI), pp. 125-134, Sept. 2014.

[12] R. J. Cole, D. F. Kripke, W. Gruen, D. J. Mullaney, and J. C. Gillin, "Automatic sleep/wake identification from wrist activity," Sleep, vol. 15 , pp. 461-469, Oct. 1992.

[13] N. Selvaraj, "Long-term remote monitoring of vital signs using a wireless patch sensor," in 2014 IEEE Healthcare Innovation Conference (HIC), pp. 83-86, Oct. 2014.

[14] M. Dontje, M. van der Wal, R. Stolk, J. Bruegemann, T. Jaarsma, P. Wijtvliet, C. van der Schans, and M. de Greef, "Daily Physical Activity in Stable Heart Failure Patients," Journal of Cardiovascular Nursing, vol. 29, no. 3, pp. 218-226, 2014.

[15] O. K. Basoglu, B. Keskin, M. S. Tasbakan, and C. Gurgun, "Effect of Semirecumbent Sleep Position on Severity of Obstructive Sleep Apnea in Patients With Heart Failure," Journal of Cardiac Failure, vol. 21, pp. 842-847, Oct. 2015.

[16] M. Boehm, K. Swedberg, M. Komajda, J. S. Borer, I. Ford, A. DubostBrama, G. Lerebours, and L. Tavazzi, "Heart rate as a risk factor in chronic heart failure (SHIFT): the association between heart rate and outcomes in a randomised placebo-controlled trial," The Lancet, vol. 376, pp. 886-894, Sept. 2010. 